\title{
A bis-vanadyl coordination complex as a 2-qubit quantum gate
}

\author{
Ivana Borilovic, ${ }^{a, \dagger}$ Pablo J. Alonso, ${ }^{b}$ Olivier Roubeau ${ }^{\mathrm{b}, *}$ and Guillem Aromí ${ }^{\mathrm{a}, \mathrm{c}, *}$
}

a Departament de Química Inorgànica i Orgànica, Universitat de Barcelona, Diagonal 645, 08028 Barcelona, Spain. E-mail:
guillem.aromi.qi.ub.es.

b Instituto de Ciencia de Materiales de Aragón (ICMA), CSIC and Universidad de Zaragoza, Plaza San Francisco s/n, 50009, Zaragoza, Spain. E-mail: roubeau@unizar.es

c Institut of Nanoscience and Nanotechnology of the University of Barcelona (IN2UB), Barcelona, Spain.

† Current address: Photon Science Institute and Department of Chemistry, University of Manchester, Oxford Road, Manchester M139PL, United Kingdom.

A new bis-hydroxyphenylpyrazolyl ligand, $\mathrm{H}_{4} \mathrm{~L}$, allows to isolate and structurally characterize vanadyl and titanyl dinuclear complexes $\left(\mathrm{Bu}_{4} \mathrm{~N}\right)_{2}\left[(\mathrm{MO})_{2}(\mathrm{HL})_{2}\right] \quad(\mathrm{M}=\mathrm{V}, \mathrm{Ti})$. The weak dipolar coupling and relatively short quantum coherence of the divanadyl anion are optimal for a 2-qubit molecular architecture proposed to implement electron-mediated nuclear quantum simulations.

One of the most pursued scientific goals is the realization of quantum computing, ${ }^{1}$ which uses the laws and resources of quantum mechanics to implement fast very complex algorithms, ${ }^{2-4}$ realize quantum simulations $^{5}$ or exploit quantum cryptography. ${ }^{6}$ This requires a two-level quantum system as the elementary unit of information (qubit) and a technology to address these qubits in logical ways and interconnect them for computation. Among the proposed systems for realizing qubits, ${ }^{7-10}$ the molecular electronic spin is especially attractive for chemists. ${ }^{11-13}$ Thus, important efforts have been made to understand the factors controlling the quantum coherence of the spin in transition metal ${ }^{14-17}$ and lanthanide coordination complexes. ${ }^{18-20}$ The realization of quantum gates requires the coherent manipulation of several inter-connected qubits. Molecules have been prepared as prototypes of 2-qubit quantum gates, either as dimers of inequivalent entangled metal ions, ${ }^{21,22}$ or as metal-based pairs of qubits with a switchable interaction. ${ }^{23,24}$ It has also been suggested to use the nuclear spin degrees of freedom as $N$-qudits (units of information of dimension $N$ ), ${ }^{25,26}$ and some schemes rely on the hyperfine interaction between the nuclear and electronic spins for the implementation of elaborate protocols, such as quantum error correction methods ${ }^{27}$ or the realization of the Grover algorithm. ${ }^{28}$ A recent report puts forward a coordination anion containing two weakly coupled vanadyl centres, each exhibiting hyperfine coupling between its electronic spin $(S=1 / 2)$ and its nuclear magnetic moment $(I=7 / 2){ }^{29}$ The characteristics of this molecule were used to simulate the theoretical implementation of a 2-qubit scheme with both qubits encoded by two $m_{l}$ states of each nuclear spin (specifically, $|0\rangle=|5 / 2\rangle$ and $|1\rangle=|7 / 2\rangle$ ). A switchable interaction between both qubits and their entanglement is ensured by the coupling between the electronic spin moments through the hyperfine coupling at each metal.
Thus, the interaction between both electronic spins makes any inversion of these spins using microwaves dependent on the nuclear spin, which allows the implementation of a conditional 2-qubit gate. The lack of a single crystal X-ray structure however limits a detailed description of this gate, while the qubit separation and associated spin-spin interaction may need to be optimized. Thus, this important development urgently requires model qugate systems allowing exact correlations of their performance with their metric parameters and also enabling to study the isolated gates inside a diamagnetic matrix.

We report here the synthesis and structure of a novel bis-hydroxyphenylpyrazolyl ligand, $\mathrm{H}_{4} \mathrm{~L}$, causing the formation of vanadyl and titanyl containing complexes $\left(\mathrm{Bu}_{4} \mathrm{~N}\right)_{2}\left[(\mathrm{VO})_{2}(\mathrm{HL})_{2}\right](1)$ and $\left(\mathrm{Bu}_{4} \mathrm{~N}\right)_{2}\left[(\mathrm{TiO})_{2}(\mathrm{HL})_{2}\right](2$ and 2'), respectively, together with a solid solution, termed 3. Thus, the potential performance of 1 as 2-qubit quantum gate has been evaluated by magnetometry and continuous and pulsed EPR.

Ligand $\mathrm{H}_{4} \mathrm{~L}$ was conceived as a possible entry into dinuclear complexes with two separate weakly interacting metals, thanks to two spaced chelating pockets (Fig. 1). It was prepared via the double ring closure of adjacent $\beta$-diketone units with hydrazine, on the precursor molecule 1,6-bis-(2-hydroxyphenyl)1,3,4,6-hexanetetraone $\left(\mathrm{H}_{4} \mathrm{~L} 1\right.$; Scheme. S1).
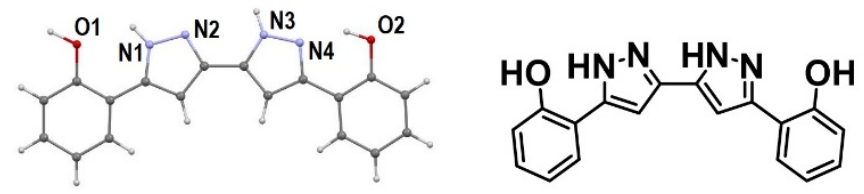

Figure 1. Ligand $\mathrm{H}_{4} \mathrm{~L}$ and representation of its molecular structure.

$\mathrm{H}_{4} \mathrm{~L} 1$ was synthesized through a Claisen condensation between two equivalents of 2-hydroxyacetophenone and one equivalent of dimethyl oxalate (Scheme S1, Figs. S1-S2). $\mathrm{H}_{4} \mathrm{~L}$ crystallizes from the reaction mixture in the monoclinic C2/c space group with half an equivalent of molecules of hydrazine, in the form of pairs of almost flat $\mathrm{H}_{4} \mathrm{~L}$ molecules disposed parallel, side-by-side, and connected through hydrogen bonds (Tables $\mathrm{S} 1$ and S3, Figs. S4-S6). $\mathrm{H}_{4} \mathrm{~L}$ was then recrystallized from acetone, deprotonated with $\mathrm{Bu}_{4} \mathrm{NOH}$ in pyridine and made to react with $\mathrm{VOSO}_{4}$ (Eq. 1), 
producing violet crystals of 1 upon diffusion of $\mathrm{Et}_{2} \mathrm{O}$ to the reaction mixture.

$2 \mathrm{H}_{4} \mathrm{~L}+6 \mathrm{Bu}_{4} \mathrm{NOH}+2 \mathrm{VOSO}_{4} \rightarrow$

$\left(\mathrm{Bu}_{4} \mathrm{~N}\right)_{2}\left[(\mathrm{VO})_{2}(\mathrm{HL})_{2}\right]+2\left(\mathrm{Bu}_{4} \mathrm{~N}\right)_{2} \mathrm{SO}_{4}+6 \mathrm{H}_{2} \mathrm{O}$

The analogous reaction using $\mathrm{TiO}(\text { acac })_{2}$ leads to the formation of polymorphs $\mathbf{2}$ and 2', as light-yellow crystals. The molecular structure of the complexes was established by SCXRD (see below). In view of this, a solid solution was obtained by dissolving in pyridine crystals of the $(\mathrm{VO})^{2+}$ and $(\mathrm{TiO})^{2+}$ complexes in the $1: 10$ molar ratio. Diffusion of $\mathrm{Et}_{2} \mathrm{O}$ into this mixture caused the crystallization of light-yellow crystals with some violet reflects of the doped system 3 , with formulation close to $\left(\mathrm{Bu}_{4} \mathrm{~N}\right)_{2}\left[(\mathrm{VO})_{2}(\mathrm{HL})_{2}\right]_{0.10}\left[(\mathrm{TiO})_{2}(\mathrm{HL})_{2}\right]_{0.90}$.

Complexes $\mathbf{1}$ and $\mathbf{2}$ crystallize in the triclinic space group $P \overline{1}$, but in different cells respectively as a pyridine solvate and unsolvated complex, while polymorph $\mathbf{2}^{\prime}$ crystalizes in the monoclinic space group $P 2_{1} / \mathrm{n}$. Interestingly, compound $\mathbf{3}$ crystallizes in the same solvated phase as $\mathbf{1}$, as if the minor component of the solution was acting as template for the mode of crystallization. The unit cell of 1 contains two crystallographically inequivalent $\left[(\mathrm{VO})_{2}(\mathrm{HL})_{2}\right]^{2-}$ complex anions, almost identical to each other. Four $\mathrm{Bu}_{4} \mathrm{~N}^{+}$ cations and nine solvate pyridine molecules complete the unit cell content, the asymmetric unit consisting of half this composition. The asymmetric unit of 2 comprises one half $\left[(\mathrm{TiO})_{2}(\mathrm{HL})_{2}\right]^{2-}$ complex and one disordered $\mathrm{Bu}_{4} \mathrm{~N}^{+}$cation, while the asymmetric unit of 2' comprises one half $\left[(\mathrm{TiO})_{2}(\mathrm{HL})_{2}\right]^{2-}$ complex and one $\mathrm{Bu}_{4} \mathrm{~N}^{+}$cation. The complexes $\left[(\mathrm{VO})_{2}(\mathrm{HL})_{2}\right]^{2-}$ and $\left[(\mathrm{TiO})_{2}(\mathrm{HL})_{2}\right]^{2-}$ in 1 and $2 / 2^{\prime}$ are very similar, both well represented by Fig. 2, and will be described together. They contain two flat $\mathrm{HL}^{3-}$ ligands, parallel and facing each other, linked by their coordination to two $\mathrm{V}(\mathrm{IV}) / \mathrm{Ti}(\mathrm{IV})$ metals. The latter are chelated at their equatorial sites and bridged by the $\mathrm{HL}^{3-}$ donors, which keep them (hereafter in the 1/2/2' format) 8.267/8.511/8.538 $\AA$ (in average for 1) apart. The coordination of all the metals is squared pyramidal by virtue of the oxo groups at one of both apical positions of each metal. Within each complex, these oxo groups are mutually trans by symmetry (i.e. pointing to opposite sides of the molecular plane). The average equatorial $\mathrm{M}-\mathrm{O}$ and $\mathrm{M}-\mathrm{N}$ bond distances are, respectively, 1.928/1.907/1.906 and 2.089/2.183/2.182 $\AA$. A larger affinity for oxygen than for nitrogen is reflected in these distances, which is more evident for Ti(IV) than for $\mathrm{V}(\mathrm{IV})$. The $\mathrm{M}=\mathrm{O}$ separations are $1.601 / 1.643 / 1.644 \AA$. The effect of the oxo group causes a separation of the metals from the equatorial planes, with distances of $0.536 / 0.561 / 0.510 \AA$. The shortest intermolecular metal-metal distance is 9.025/7.453/7.188 $\AA$. Despite the planarity of the complex anions and their aromatic rings, the lattices of $\mathbf{1}$ and 2 do not show $\pi \cdots \pi$ interactions. In 1, the complexes are arranged with the same orientation, approximately as layers that alternate with layers of $\mathrm{Bu}_{4} \mathrm{~N}^{+}$cations, with pyridine molecules accommodated within the voids (Fig. S7). In 2/2', the structure is much more compact, given the lack of pyridine, and in 2' the complexes exhibit two orientations (Fig. S8). The arrangement of the molecules in $\mathbf{3}$ is analogous to the organization in $\mathbf{1 .}$


Figure 2. Top and side views of the molecular structure of the anion $\left[(\mathrm{VO})_{2}(\mathrm{HL})_{2}\right]^{2-}$ of 1 . Unique heteroatoms labelled. $\mathrm{C}$ atoms in grey. Only $\mathrm{H}$ atoms on $\mathrm{N}$ atoms shown.

To evaluate the potential of the vanadyl pairs in $\left[(\mathrm{VO})_{2}(\mathrm{HL})_{2}\right]^{2-}$ complex anion as a 2-qubit architecture, the bulk magnetic properties of 1 were first examined. The magnetization vs. field data at $2 \mathrm{~K}$ follows the Brillouin function for 2 isolated $S=1 / 2$ spins with $g=$ 1.98 (Fig. S9), while the temperature dependence of $\chi T, \chi$ being the molar magnetic susceptibility, is well reproduced with a Curie-Weiss model with $C=0.7398$ $\mathrm{cm}^{3} \mathrm{~mol}^{-1}(\mathrm{~g}=1.973)$ and $\theta / \mathrm{k}_{\mathrm{B}}=-0.07 \mathrm{~K}\left(\theta=-0.048 \mathrm{~cm}^{-}\right.$ 1 , Fig. S9), altogether pointing a paramagnet with only very weak antiferromagnetic interactions. Considering the similar intra- and inter-molecular $\mathrm{V} \cdots \mathrm{V}$ separations and the absence of clear exchange-coupling pathway(s), the most likely origin of these is throughspace dipolar interaction. On the other hand, the solidstate continuous-wave (CW) EPR spectra of 1 and 3 are quite similar, except for the sharper lines in the case of magnetically dilute 3 (Figure S10). The spectrum of 3 is consistent with hyperfine coupled vanadyl species that are in addition weakly interacting with each other. This supports the presence of weak magnetic interactions within the molecular $\left[(\mathrm{VO})_{2}(\mathrm{HL})_{2}\right]^{2-}$ complex, one of the requirements for the proposed 2qubit scheme. $^{29}$

The spin dynamics of the $\left[(\mathrm{VO})_{2}(\mathrm{HL})_{2}\right]^{2-}$ species were first evaluated determining the spin-lattice relaxation time $T_{1}{ }^{\text {ac }}$ of 1 by ac susceptibility measurements. While no out-of-phase component is detected in zero-field at $1.8 \mathrm{~K}$, a $d c$ field as low as $25 \mathrm{mT}$ brings the maximum of the out-of-phase component, which defines the magnetization relaxation characteristic time, in the experimental frequency window. At low fields, $T_{1}^{\text {ac }}$ increases to reach ca. $12 \mathrm{~ms}$ at $0.75 \mathrm{~T}$, and then decreases markedly above $1 \mathrm{~T}$ (Fig. 3 left). The lowfield increase approximates to $B^{2}$. It is caused by the growing $d c$ field overcoming spin-spin and spin-nucleus interactions, at the origin of the two-phonon spin relaxation processes by breaking the degeneracy of the Kramers doublet. 

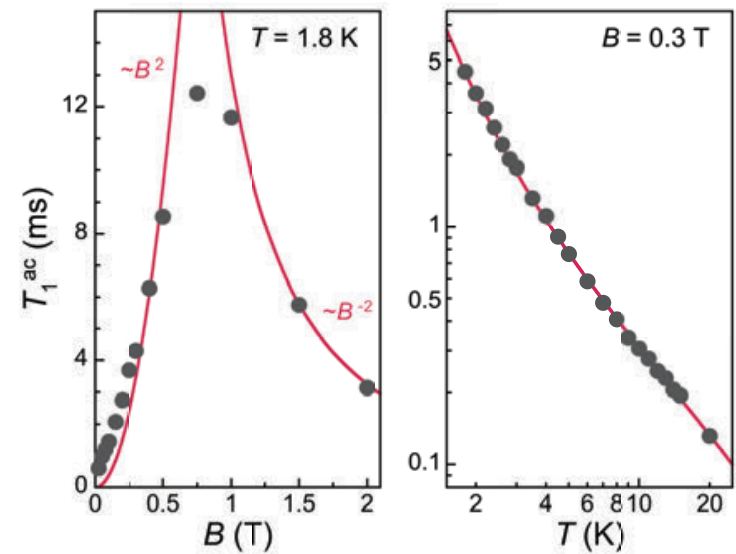

Figure 3. Spin-lattice relaxation time $T_{1}{ }^{\text {ac }}$ for 1 as a function of the $d c$ magnetic field $B$ at $1.8 \mathrm{~K}$ (left) and of temperature at $0.3 \mathrm{~T}$ (right). Red lines are respectively $B^{2}$ and $B^{-2}$ dependences (left) and a fit of the data to $T_{1}{ }^{a c-1}=a B^{2} T+$ $b \Delta /[\exp (\Delta / T)-1]+c T^{m}$ (right)

The fast decrease at higher fields corresponds to the direct relaxation process, typically dominant at high fields, and expected to be $\approx B^{-4}$ for a Kramers system. Here, the dependence is closer to $B^{-2}$, indicating phonon bottleneck effects, expected for magnetically concentrated solids. ${ }^{30}$ The temperature dependence of $T_{1}{ }^{\text {ac }}$ (Fig. 3 right) exhibits the expected decrease due to thermally-activated relaxation processes, albeit fairly weak (close to $\approx T^{-1.3}$ ), confirming the presence of phonon bottleneck effects. ${ }^{30,31}$ A good simulation of it was obtained considering direct, Orbach and Raman processes through the expression $\left(T_{1}{ }^{\mathrm{ac}}\right)^{-1}=\mathrm{a} B^{2} T+$ $b \Delta /[\exp (\Delta / T)-1]+c T^{n}$, with $a=343(50) \mathrm{s}^{-1} \mathrm{~K}^{-1}, b=22(2)$ $\mathrm{s}^{-1} \mathrm{~K}^{-3}, c=0.08(2) \mathrm{s}^{-1} \mathrm{~K}^{-3}$, and the Orbach gap and Raman exponent fixed at $\Delta=4 \mathrm{~K}$ and $\mathrm{n}=3$, respectively. Both direct and Raman processes are found to be of the same order as in the monometallic $\left[\mathrm{VO}(\mathrm{dbm})_{2}\right]$ molecule. ${ }^{15}$ The Orbach-type relaxation can reasonably be ascribed to interactions among the vanadyl spins, also surfacing in this temperature range in the $\chi T$ vs. $T$ data, and providing the necessary excited state. Overall, the fairly long spin relaxation times and field and temperature dependences in 1 are in line with those of other $S=1 / 2$ molecular qubits. ${ }^{15,32}$

Pulsed-EPR was used to evaluate the presence of quantum coherence in $\mathbf{3}$. At low temperature, a rather strong electron spin-echo (ESE) signal is detected at any field in the range 280-430, thus consistent with the CW-EPR spectrum, and confirming that the vanadyl pairs exhibit measurable quantum coherence. Using a 2-pulse Hahn echo detection, a well-resolved ESEdetected EPR spectrum was determined for 3 at $6 \mathrm{~K}$. The latter is again characteristic of a weakly-interacting system with a complex set of lines likely resulting from the combination of splitting processes caused by both hyperfine spin-nuclei $\left(S=1 / 2\right.$ and $I=7 / 2$ for ${ }^{51} \mathrm{~V}^{\text {IV }}$, with $99.76 \%$ natural abundance) and spin-spin interactions (Fig. 4). Attempts at simulating the spectrum were done considering the later coupling to be either purely dipolar, exchange-coupled isotropic or planar (see ESI). Neither exchange-coupled models reproduce the features of the experimental spectrum (Figs. S13-S14),

and the best simulation is obtained using a dipolar interaction $J_{D} / k_{B}=30 \mathrm{mK}\left(J_{D}=0.021 \mathrm{~cm}^{-1}\right.$, Figs. 4 and $\mathrm{S} 15)$, confirming the assumption that the dipolar interaction is the most relevant in $\mathbf{1}$. With the used formalism (see ESI), the positive sign of $J_{D}$ corresponds to an antiferromagnetic interaction, in agreement with the Curie-Weiss simulation of the susceptibility data. This in turn indicates that the axes of quantization of the vanadyl spins are not along the $\mathrm{V} \cdots \mathrm{V}$ axis, but rather along the $\mathrm{V}=\mathrm{O}$ bonds. The estimated interaction is much larger than that calculated for the observed $\mathrm{V} \cdots \mathrm{V}$ separation through the point-dipole approximation, ca. $4.4 \mathrm{mK}$. Such strong deviations have been shown to arise from even modest spin delocalization in spin pairs connected by unsaturated linkers, ${ }^{33}$ as is the case in 1 . Interestingly, our estimation is in line with that calculated for a radical pair with a similar separation. ${ }^{33}$ The interaction in $\mathbf{1}$ is also about one order of magnitude larger than in the divanadyl anion originally proposed for a 2-qubit architecture, $\left[\mathrm{PPh}_{4}\right]\left[(\mathrm{VO})_{2}(\mathrm{~L} 2)_{2}\right]$ (4, $\mathrm{H}_{4} \mathrm{~L} 2=$ dicatechol Schiff base ligand). ${ }^{29}$ The difference can reasonably be ascribed to the change in $\mathrm{V} \cdots \mathrm{V}$ separation, shorter by about $2 \AA$ in $1 / 3$ than in 4 . Altogether, this shows the ability to tune the inter-qubit interaction by designing the linking ligand.

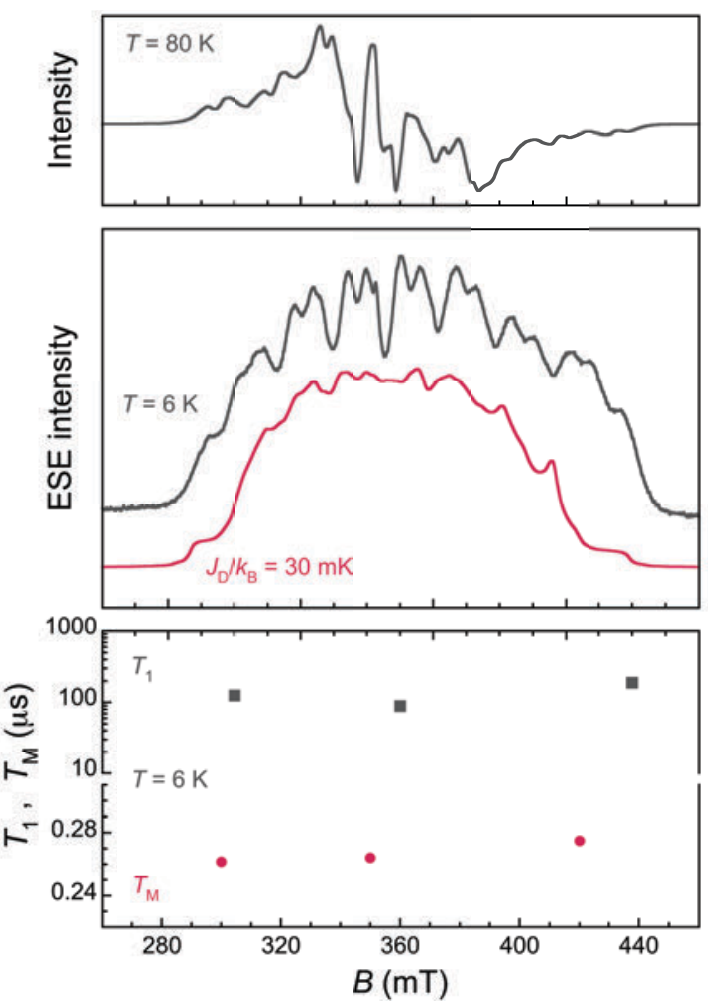

Figure 4. Top: X-band CW-EPR spectrum of 3 at $80 \mathrm{~K}$. Middle: ESE-detected 2p EPR spectrum of 3 at $6 \mathrm{~K}$ (top grey trace, average of spectra with $\tau=140$ and $200 \mathrm{~ns}$ ) and its simulation (bottom red trace) considering two equivalent axial vanadyl coupled through a dipolar interaction with $J_{D} / k_{B}=30$ $\mathrm{mK}\left(J_{D}=0.021 \mathrm{~cm}^{-1}\right.$, see ESI). Bottom: mean longitudinal relaxation time $T_{1}$ and phase memory time $T_{\mathrm{M}}$ for 3 at $6 \mathrm{~K}$ (see ESI).

The spin-lattice relaxation time $T_{1}$ and the phase memory time $T_{\mathrm{M}}$ at $6 \mathrm{~K}$ of 3 were estimated at three different magnetic fields through inversion recovery and echo decay experiments, respectively (Fig. 4, see ESI 
for details). The mean spin-lattice relaxation is found to be of the order of $90-190 \mu s$ in 3 , in reasonable agreement with values derived by ac susceptibility for 1 . $T_{\mathrm{M}}$ of the vanadyl spins in $\mathbf{3}$ appears to be basically constant at 0.26-0.27 $\mu$ s over the field range studied, thus about only four times shorter than for the dilute frozen solution of $4 .^{29}$ This remains remarkable considering the concentrated nature of 3 , with a dilution of only about $10 \%$, and the additional source of decoherence associated with the close proximity of the two electronic spins in the $\left[(\mathrm{VO})_{2}(\mathrm{HL})_{2}\right]^{2-}$ complex. It must be emphasized here that in the proposed scheme, the electron spin is not embodying the qubit, in fact, its function requires a relatively fast decoherence. ${ }^{29}$ Indeed, the dephasing of the electronic spins is only relevant for the electron-mediated implementation of fast ( $\leq 150 \mathrm{~ns}$ ) 2-qubit gates, so that the dephasing time in 3 appears to be optimal.

In conclusion, through the adequate design of a bis-chelating ligand, we have prepared and structurally characterized a complex anion featuring two vanadyl moieties in the adequate separation to favour a weak though significant through-space dipolar interaction, necessary to realize a proposed 2-qubit molecular architecture. The spin dephasing time of the divanadyl complex anion has been determined in the solid-state thanks to the ability to dilute it in its titanyl diamagnetic analogue and found to be optimal for the implementation of the proposed computation scheme.

This research was supported by Spanish MINECO (CTQ2015-68370-P, PGC2018-098630-B-I00, MAT2017-86826-R, CTQ2015-64486-R), the Aragón government (E31_17R) and EU quantERA (SUMO, PCl2018-093106, PCl2018-093116), and used resources of ALBA synchrotron and of the Advanced Light Source, which is a DOE Office of Science User Facility under contract no. DEAC02-05CH11231. G.A. thanks the Generalitat de Catalunya for the prize ICREA Academia 2018. Authors are grateful to Dr. J. I. Martínez for repeated EPR measurements.

\section{Notes and references}

${ }^{1}$ M. A. Nielsen and I. L. Chuang, Quantum Computation and Quantum Information, Cambridge University Press, 2000.

2 D. Deutsch and R. Jozsa, Proc. Roy. Soc. London Series aMath. Phys. Eng. Sci., 1992, 439, 553-558.

${ }^{3}$ P. W. Shor, Siam J. Computing, 1997, 26, 1484-1509.

${ }^{4}$ L. K. Grover, Phys. Rev. Lett., 1997, 79, 325-328.

5 I. M. Georgescu, S. Ashhab and F. Nori, Rev. Mod. Phys., 2014, 86, 153-185.

${ }^{6}$ N. Lütkenhaus and A. J. Shields, New J. Phys., 2009, 11, 045005.

7 T. D. Ladd, F. Jelezko, R. Laflamme, Y. Nakamura, C. Monroe and J. L. O'Brien, Nature, 2010, 464, 45-53.

${ }^{8}$ M. H. Devoret and R. J. Schoelkopf, Science, 2013, 339, 1169.

${ }^{9}$ C. Monroe and J. Kim, Science, 2013, 339, 1164-1169.

${ }^{10}$ T. H. Taminiau, J. Cramer, T. van der Sar, V. V. Dobrovitski and R. Hanson, Nat. Nanotechnol., 2014, 9, 171-176.
${ }^{11}$ A. Ardavan, O. Rival, J. J. L. Morton, S. J. Blundell, A. M. Tyryshkin, G. A. Timco and R. E. P. Winpenny, Phys. Rev. Lett., 2007, 98, 057201.

${ }^{12}$ G. Aromí, D. Aguilà, P. Gamez, F. Luis and O. Roubeau, Chem. Soc. Rev., 2012, 41, 537-546.

${ }^{13}$ A. Gaita-Ariño, F. Luis, S. Hill and E. Coronado, Nature Chem., 2019, 11, 301-309.

${ }^{14}$ K. Bader, D. Dengler, S. Lenz, B. Endeward, S.-D. Jiang, P. Neugebauer and J. van Slageren, Nature Commun., 2014, 5, 5304.

${ }^{15}$ M. Atzori, E. Morra, L. Tesi, A. Albino, M. Chiesa, L. Sorace and R. Sessoli, J. Am. Chem. Soc., 2016, 138, 11234-11244.

${ }^{16}$ M. S. Fataftah, J. M. Zadrozny, S. C. Coste, M. J. Graham, D. M. Rogers and D. E. Freedman, J. Am. Chem. Soc., 2016, 138, 1344-1348

17 M. S. Fataftah, M. D. Krzyniak, B. Vlaisavljevich, M. R. Wasielewski, J. M. Zadrozny and D. E. Freedman, Chem. Sci., 2019, 10, 6707-6714.

${ }^{18}$ M. J. Martínez-Pérez, S. Cardona-Serra, C. Schlegel, F. Moro, P. J. Alonso, H. Prima-Garcia, J. M. Clemente-Juan, M. Evangelisti, A. Gaita-Arino, J. Sesé, J. van Slageren, E. Coronado and F. Luis, Phys. Rev. Lett., 2012, 108, 247213.

${ }^{19}$ M. D. Jenkins, Y. Duan, B. Diosdado, J. J. Garcia-Ripoll, A. Gaita-Arino, C. Gimenez-Saiz, P. J. Alonso, E. Coronado and F. Luis, Phys. Rev. B, 2017, 95, 8.

${ }^{20}$ K. S. Pedersen, A.-M. Ariciu, S. McAdams, H. Weihe, J. Bendix, F. Tuna and S. Piligkos, J. Am. Chem. Soc., 2016, 138, 5801-5804

21 D. Aguilà, L. A. Barrios, V. Velasco, O. Roubeau, A. Repollés, P. J. Alonso, J. Sesé, S. J. Teat, F. Luis and G. Aromí, J. Am. Chem. Soc., 2014, 136, 14215-14222.

${ }^{22}$ F. Luis, A. Repollés, M. J. Martínez-Pérez, D. Aguilà, O. Roubeau, D. Zueco, P. J. Alonso, M. Evangelisti, A. Camón, J. Sesé, L. A. Barrios and G. Aromí, Phys. Rev. Lett., 2011, 107.

${ }^{23}$ J. Salinas-Uber, M. Estrader, J. Garcia, P. Lloyd-Williams, A. Sadurni, D. Dengler, J. van Slageren, N. F. Chilton, O. Roubeau, S. J. Teat, J. Ribas-Ariño and G. Aromí, Chem., Eur. J., 2017, 23, 13648-13659.

24 J. Ferrando-Soria, E. Moreno Pineda, A. Chiesa, A. Fernandez, S. A. Magee, S. Carretta, P. Santini, I. J. VitoricaYrezabal, F. Tuna, G. A. Timco, E. J. L. Mclnnes and R. E. P. Winpenny, Nature Commun., 2016, 7, 11377.

25 E. Moreno-Pineda, M. Damjanović, O. Fuhr, W. Wernsdorfer and M. Ruben, Angew. Chem. Int. Ed., 2017, 56, 9915-9919.

${ }^{26}$ S. Thiele, F. Balestro, R. Ballou, S. Klyatskaya, M. Ruben and W. Wernsdorfer, Science, 2014, 344, 1135-1138.

${ }^{27}$ R. Hussain, G. Allodi, A. Chiesa, E. Garlatti, D. Mitcov, A. Konstantatos, K. S. Pedersen, R. De Renzi, S. Piligkos and S. Carretta, J. Am. Chem. Soc., 2018, 140, 9814-9818.

${ }_{28}$ C. Godfrin, A. Ferhat, R. Ballou, S. Klyatskaya, M. Ruben, W. Wernsdorfer and F. Balestro, Phys. Rev. Lett., 2017, 119, 187702

${ }^{29}$ M. Atzori, A. Chiesa, E. Morra, M. Chiesa, L. Sorace, S. Carretta and R. Sessoli, Chem. Sci., 2018, 9, 6183-6192.

${ }^{30} \mathrm{~J}$. Soeteman, A. J. van Duyneveldt, C. L. M. Pouw and W. Breur, Physica, 1973, 66, 63-69.

${ }^{31}$ S. Gómez-Coca, A. Urtizberea, E. Cremades, P. J. Alonso, A. Camón, E. Ruiz and F. Luis, Nature Commun., 2014, 5, 4300.

${ }^{32}$ A. Urtizberea, E. Natividad, P. J. Alonso, M. A. Andrés, I. Gascón, M. Goldmann and O. Roubeau, Adv. Funct. Mater., 2018, 28, 1801695

${ }^{33}$ C. Riplinger, J. P. Y. Kao, G. M. Rosen, V. Kathirvelu, G. R. Eaton, S. S. Eaton, A. Kutateladze and F. Neese, J. Am. Chem. Soc., 2009, 131, 10092-10106 